\title{
Doubly Degenerate Parabolic Equations with Variable Nonlinearity II: Blow-up and Extinction in a Finite Time
}

\author{
S. N. Antontsev \\ CMAF, University of Lisbon, Portugal \\ S. I. Shmarev* \\ University of Oviedo, Oviedo, Spain
}

Keywords: Nonlinear parabolic equations, double nonlinearity, nonstandard growth conditions, blow-up, extinction, decay rates,

2010 MSC: 35K55, 35K65, 35K67

\section{Introduction}

The paper is devoted to the study of the qualitative properties of energy solutions of the Dirichlet problem for the doubly nonlinear anisotropic parabolic equation with variable nonlinearity

$$
\left\{\begin{array}{l}
\frac{d}{d t}\left(|v|^{m(z)} \operatorname{sign} v\right)=\sum_{i=1}^{n} D_{i}\left(a_{i}(z)\left|D_{i} v\right|^{p_{i}(z)-2} D_{i} v\right)+b(z)|v|^{\sigma(z)-2} v+g \text { in } Q_{T} \\
v=0 \text { on } \Gamma_{T} \\
v(x, 0)=v_{0}(x) \text { in } \Omega
\end{array}\right.
$$

where $Q_{T}=\Omega \times(0, T), \Omega \subset \mathbb{R}^{n}$ is a bounded domain with Lipschitz-continuous boundary $\partial \Omega$, $\Gamma_{T}=\partial \Omega \times(0, T)$ is the lateral boundary of the cylinder $Q_{T}$. The exponents of nonlinearity $m(z), p_{i}(z)$ and $\sigma(z)$ are given functions of the argument $z=(x, t) \in Q_{T}$.

The total derivative with respect to $t$ is calculated according to the rule

$$
\frac{d}{d t} \psi(v, m)=\psi_{v}(v, m) v_{t}+\psi_{m}(v, m) m_{t}
$$

which allows one to rewrite equation (1.1) in the formally equivalent form

$$
m|v|^{m-1} v_{t}=\sum_{i=1}^{n} D_{i}\left(a_{i}\left|D_{i} v\right|^{p_{i}-2} D_{i} v\right)+b|v|^{\sigma-2} v-|v|^{m-1} v \ln |v| m_{t}+g .
$$

\footnotetext{
* Corresponding author

Email addresses: anton@ptmat.fc.ul.pt, antontsevsn@mail.ru (S. N. Antontsev), shmarev@uniovi.es (S. I. Shmarev)
} 
This equation can degenerate or become singular at the points where $\left|D_{i} v\right|$ and/or $v$ vanish or become infinite. Moreover, the behavior of the solution may depend crucially on the sign of $m_{t}(x, t)$ in the logarithmic nonlinear term on the right-hand side.

The study of problem (1.1) was initiated in [1,7]. It was shown that under certain conditions on the data problem (1.1) admits a bounded solution understood in the sense of Definition 2.1 below. In dependence on the properties of the coefficients and the variable exponents of nonlinearity, the existence result proved in $[1,7]$ is local or global in time (see Theorem 2.1 below). The maximal interval of existence is defined as the time interval where the solution remains bounded.

In the present paper we derive the conditions on the data that ensure the presence of the following effects.

- Finite time blow-up: there exists a finite moment $t^{*}$ such that

$$
\Sigma(t) \equiv \int_{\Omega}|v|^{m(x, t)+1} d x \rightarrow \infty \quad \text { as } t \rightarrow t^{*},
$$

(which implies that $\|v(\cdot, t)\|_{\infty, \Omega}$ becomes infinite at the same moment).

- Extinction in a finite time: there is a finite $t_{*}>0$ such that $\Sigma(t) \equiv 0$ for all $t \geq t_{*}$. In particular, this effect may take place even in the case when the equation eventually becomes linear. This happens if $m \rightarrow 1, \sigma \rightarrow 2$ and $p_{i} \rightarrow 2$ as $t \rightarrow \infty$ and the rates of convergence satisfy some additional conditions.

- In case that the solution exists for all times but doesn't vanish in a finite time, we estimate the rate of growth or decay of $\Sigma(t)$ as $t \rightarrow \infty$.

Examples of conditions on the data which provide the above-described effects can be furnished by the model equation

$$
\frac{d}{d t}\left(|v|^{m(t)-1} v\right)=\left(\left|v_{x}\right|^{p(t)-2} v_{x}\right)_{x}+b|v|^{\sigma(t)-1} v, \quad(x, t) \in(0,1) \times(0, T],
$$

with the constant coefficient $b$ and the independent of $x$ nonlinearity exponents $m(t)>0$, $p(t)>1$ and $\sigma(t)>1$. In this simple case

- if $b>0, \sigma(t)>\max \{m(t)+1, p(t)\}, m^{\prime}(t) \in[-\mu, 0]$ with some constant $\mu>0$, and if

$$
\int_{0}^{1}\left(\frac{1}{p(0)}\left|v_{0}^{\prime}\right|^{p(0)}-\frac{1}{\sigma(0)}|v|^{\sigma(0)}\right) d x<0,
$$

then every solution blows-up for the sufficiently small $\mu$;

- if $b=0, m^{\prime}(t) \in[0, \mu]$ and $\frac{m(t) p(t)}{m(t)+1}<1$, then the solution vanishes in a finite time, provided that $\mu$ is sufficiently small; 
- if $b<0, m^{\prime}(t) \in[0, \mu]$ and $\frac{1}{\sigma(t)}+\frac{m(t)}{p(t)}>1$, then the solution vanishes in a finite time if $\mu$ is sufficiently small;

The properties of finite time vanishing and blow-up are well-studied by now for the solutions of nonlinear parabolic equation of the type (1.1) with constant exponent of nonlinearity. A thorough discussion of these issues and an extensive bibliography can be found in the monographs and surveys $[15,12,2,11,17]$. For equations with variable nonlinearity, which degenerate or become singular either with respect to the solution (the case $p_{i} \equiv 2$ ), or with respect to the gradient (the case $m \equiv 1$ ), these effects were studied in papers $[3,4,5,6]$. The decay rates for solutions of equations of the type (1.1) with constant exponents of nonlinearity can be found in [13], the case of equation (1.1) with $m=1$ and variable $p_{i}$ and $\sigma$ was partially studied in [6].

The absence of exact solutions (or sub/supersolutions) of equation (1.1) with the anisotropic diffusion part or with the time-dependent nonlinearity exponents prevents one from using the methods based on comparison. The results below are obtained by means of the study of the differential inequalities for suitable energy functions associated with the solution. In the case that the exponents of nonlinearity are constant or time-independent we recover some of the results available in the literature for doubly nonlinear equations (see, e.g., [8]).

Organization of the paper. In the next auxiliary section we introduce the spaces of Lebesgue and Sobolev with variable exponents and recall the basic properties of the functions from these spaces. We introduce the notion of strong solution of problem (1.1) and cite the existence theorem proved in [7]. We also recall the energy identity for such solutions, which happens to be the main tool in the study of the localization and blow-up properties.

In Section 3 we study equation (1.1) in the case $b(z)>0$. We derive the ordinary differential inequality for the energy function $\Sigma(t)$ and prove that under certain conditions on the data every function satisfying this inequality blows-up in a finite time.

Section 4 is devoted to derivation of the embedding inequalities in anisotropic Sobolev spaces with variable exponents. Using these inequalities we derive the differential inequality for the energy function $\Sigma(t)$ in the case $b(z) \leq 0$. The study of the differential inequality is given in Section 6: it is shown that under suitable conditions on the initial data every function satisfying this inequality vanishes in a finite time. The concluding Section 7 is devoted to the study of the decay or growth rates of the solutions as $t \rightarrow \infty$.

The specific form of the main energy identity (2.14) allows for an easy extension of the results concerning the finite-time extinction and the large-time behavior to the equations with the lower-order terms of critical growth:

$$
\frac{d}{d t}\left(|v|^{m(z)-1} v\right)=\sum_{i=1}^{n} D_{i}\left(a_{i}\left|D_{i} v\right|^{p_{i}(z)-2} D_{i} v\right)+c(z)|v|^{m(z)-1} v+b(z)|v|^{\sigma(z)-2} v+g,
$$

where $b(z) \leq 0$ and $c(z) \leq \gamma(t)$ with a bounded function $\gamma(t)$. 


\section{Function spaces, existence theorem and energy estimates}

\subsection{Function spaces}

We begin with recalling some known facts from the theory of the Sobolev spaces with variable exponent. The reader is referred to the monograph [9] for the detailed exposition of this theory.

Let $L^{p(\cdot)}(\Omega)$ be the set of measurable functions $f(x)$ on $\Omega$ such that

$$
A_{p(\cdot)}(f)=\int_{\Omega}|f(x)|^{p(x)} d x<\infty .
$$

The set $L^{p(\cdot)}(\Omega)$ equipped with the Luxemburg norm

$$
\|f\|_{p(\cdot), \Omega} \equiv\|f\|_{L^{p(\cdot)}(\Omega)}=\inf \left\{\lambda>0: A_{p(\cdot)}(f / \lambda) \leq 1\right\}
$$

becomes a Banach space. The Banach space $W_{0}^{1, p(\cdot)}(\Omega)$ with $p(x) \in\left[p^{-}, p^{+}\right] \subset(1, \infty)$ is defined by

$$
\left\{\begin{array}{l}
W_{0}^{1, p(\cdot)}(\Omega)=\left\{u \in L^{p(\cdot)}(\Omega):|\nabla u|^{p(x)} \in L^{1}(\Omega), u=0 \text { on } \partial \Omega\right\} \\
\|u\|_{W_{0}^{1, p(\cdot)}(\Omega)}=\|\nabla u\|_{p(\cdot), \Omega} .
\end{array}\right.
$$

Let us indicate the basic properties of the spaces $L^{p(\cdot)}(\Omega)$ and $W_{0}^{1, p(\cdot)}(\Omega)$ used in the rest of this paper.

- It follows directly from the definition of the norm that

$$
\min \left(\|f\|_{p(\cdot)}^{p^{-}},\|f\|_{p(\cdot)}^{p^{+}}\right) \leq A_{p(\cdot)}(f) \leq \max \left(\|f\|_{p(\cdot)}^{p^{-}},\|f\|_{p(\cdot)}^{p^{+}}\right) .
$$

- Hölder's inequality. For all $f \in L^{p(\cdot)}(\Omega), g \in L^{p^{\prime}(\cdot)}(\Omega)$ with

$$
p(x) \in(1, \infty), \quad p^{\prime}(x)=\frac{p(x)}{p(x)-1}, \quad p^{-}=\inf _{\Omega} p(x), \quad\left(p^{\prime}\right)^{-}=\inf _{\Omega} p^{\prime}(x),
$$

the following inequality holds:

$$
\int_{\Omega}|f g| d x \leq\left(\frac{1}{p^{-}}+\frac{1}{\left(p^{\prime}\right)^{-}}\right)\|f\|_{p(\cdot)}\|g\|_{p^{\prime}(\cdot)} \leq 2\|f\|_{p(\cdot)}\|g\|_{p^{\prime}(\cdot)} .
$$

Let $m(z)>0$ and $p_{i}(z)>1, i=1, \ldots, n$, be given functions continuous in $\bar{Q}_{T}$. For every fixed $t \in[0, T]$ we introduce the Banach space

$$
\begin{aligned}
& \mathbf{V}_{t}(\Omega)=\left\{u(x) \in L^{m(\cdot, t)+1}(\Omega) \cap W_{0}^{1,1}(\Omega):\left|D_{i} u(x)\right|^{p_{i}(x, t)} \in L^{1}(\Omega)\right\} \\
& \|u\|_{\mathbf{V}_{t}(\Omega)}=\|u\|_{m(\cdot, t)+1, \Omega}+\sum_{i}\left\|D_{i} u\right\|_{p_{i}(\cdot, t), \Omega} .
\end{aligned}
$$

By $\mathbf{W}\left(Q_{T}\right)$ we denote the Banach space 


$$
\begin{aligned}
& \mathbf{W}\left(Q_{T}\right)=\left\{|u|^{m(z)+1} \in L^{1}\left(Q_{T}\right):\left|D_{i} u\right|^{p_{i}(z)} \in L^{1}\left(Q_{T}\right), u(\cdot, t) \in \mathbf{V}_{t}(\Omega) \text { for a.e. } t \in(0, T)\right\}, \\
& \|u\|_{\mathbf{W}\left(Q_{T}\right)}=\sum_{i}\left\|D_{i} u\right\|_{p_{i}(\cdot, \cdot), Q_{T}}+\|u\|_{m(\cdot, \cdot)+1, Q_{T}} .
\end{aligned}
$$

\subsection{Existence of strong solutions}

The solution of problem (1.1) is understood as follows:

Definition 2.1. A function $v(z)$ is called Strong Solution of problem (1.1) if

1. $v \in L^{\infty}\left(Q_{T}\right) \cap \mathbf{W}\left(Q_{T}\right),|v|^{m(z)-1} v_{t}^{2} \in L^{1}\left(Q_{T}\right)$ and $\left|D_{i} v\right|^{p_{i}(z)} \in L^{\infty}\left(0, T ; L^{1}(\Omega)\right)$,

2. for every test-function $\phi \in \mathbf{W}\left(Q_{T}\right)$

$$
\begin{aligned}
\int_{Q_{T}} & {\left[\phi \frac{d}{d t}\left(|v|^{m-1} v\right)+\sum_{i=1}^{n} a_{i}\left|D_{i} v\right|^{p_{i}-2} D_{i} v \cdot D_{i} \phi\right] d z } \\
= & \int_{Q_{T}} b|v|^{\sigma-2} v \phi d z+\int_{Q_{T}} g \phi d z,
\end{aligned}
$$

3. for every $\psi(x) \in C_{0}^{\infty}(\Omega)$

$$
\int_{\Omega} \psi(x)\left(|v|^{m(z)-1} v-\left|v_{0}\right|^{m_{0}-1} v_{0}\right) d x \rightarrow 0 \quad \text { as } t \rightarrow 0, \quad m_{0}=m(x, 0) .
$$

Let $\phi(x, t) \in C^{0}\left(\bar{Q}_{T}\right)$. Throughout the rest of the text we use the notations

$$
\begin{array}{ll}
\phi^{+}(t)=\sup _{\Omega} \phi(x, t), & \phi^{-}(t)=\inf _{\Omega} \phi(x, t), \\
\phi^{+}=\sup _{Q_{T}} \phi(x, t), & \phi^{-}=\inf _{Q_{T}} \phi(x, t) .
\end{array}
$$

Let us assume that the coefficients of equation (1.1) and the exponents of nonlinearity are subject to the following conditions:

$$
\begin{aligned}
& \left\{\begin{array}{c}
\text { there exist positive constants } a^{ \pm}, b^{ \pm} p^{ \pm}, m^{ \pm}, \sigma^{ \pm} \text {such that } \\
0<a^{-} \leq a_{i}(z) \leq a^{+}, \quad-b^{-} \leq b(z) \leq b^{+}, \\
1<p^{-} \leq p_{i}(z) \leq p^{+}, \quad 1<\sigma^{-} \leq \sigma(z) \leq \sigma^{+}, \quad 0<m^{-} \leq m(z) \leq m^{+},
\end{array}\right. \\
& \left\{\begin{array}{l}
m(z), \sigma(z) \in C^{0}\left(\bar{Q}_{T}\right), \quad p_{i}(z) \text { satisfy the log-continuity condition } \\
\forall z_{1}, z_{2} \in \bar{Q}_{T} \quad\left|p_{i}\left(z_{1}\right)-p_{i}\left(z_{2}\right)\right| \leq \omega\left(\left|z_{1}-z_{2}\right|\right), \quad \varlimsup_{\tau \rightarrow 0+} \omega(\tau) \ln \frac{1}{\tau}=C<\infty,
\end{array}\right. \\
& m_{t} \in L^{\infty}\left(Q_{T}\right), \quad \exists \mu=\text { const }>0: \quad\left|m_{t}\right| \leq \mu \text { a.e. in } Q_{T}, \\
& \left\{\begin{array}{l}
0 \leq-p_{i t}(z) \leq C_{p}, \quad 0 \leq b(z) \sigma_{t}(z) \leq C_{\sigma}, \\
0 \leq-a_{i t}(z) \leq C_{a}, \quad 0 \leq b_{t}(z) \leq C_{b},
\end{array}\right.
\end{aligned}
$$

with nonnegative constants $C_{a}, C_{b}, C_{\sigma}, C_{p}$. 
Theorem 2.1 (Th. 3.1, [7]). Let the exponents $m(z), \sigma(z), p_{i}(z)$ satisfy conditions (2.6), (2.7), (2.9) and let $m_{t} \geq 0$ a.e. in $Q_{T}$. Let us assume that there exists a constant $\alpha>0$ such that if $p^{-}(t)<n$ for some $t \in[0, T]$, then

$$
m^{+}(t)+1+\alpha<\frac{n p^{-}(t)}{n-p^{-}(t)}, \quad \text { where } \quad \frac{1}{p^{-}(t)}=\frac{1}{n} \sum_{i=1}^{n} \frac{1}{p_{i}^{-}(t)} .
$$

Assume that $m(z)$ satisfies the oscillation condition

$$
\frac{m^{+}-1}{2}<\min \left\{m^{-},\left(1-\frac{1}{p^{-}}\right)\left(m^{-}+1\right)\right\} \quad \text { if } m^{+}>1 \text { and } \sum_{i=1}^{n} \frac{1}{p_{i}^{-}}>1
$$

Then for every $v_{0} \in L^{\infty}(\Omega) \cap \mathbf{V}_{0}(\Omega)$ and every $g \in L^{\infty}\left(Q_{T}\right)$ with $\left|g_{t}(z)\right|^{1+\frac{1}{m(z)}} \in L^{1}\left(Q_{T}\right)$ problem (1.1) has at least one strong solution in a cylinder $Q_{T^{*}}$,

$$
T^{*}=\sup \left\{\theta:\|v(t)\|_{\infty, \Omega}<\infty \forall t \in(0, \theta)\right\} .
$$

Moreover, for small $\tau$ the solution satisfies the estimate

$$
\|v(t)\|_{\infty, \Omega} \leq\left(1+\left\|v_{0}\right\|_{\infty, \Omega}\right) \mathrm{e}^{A t}, \quad t \in[0, \tau]
$$

with a constant $A$ independent of $v$. If either $\sup _{Q_{T}}(\sigma(z)-m(z)) \leq 1$, or $b^{+} \leq 0$, then estimate (2.12) remains true for all $T>0$, the constructed solution exists globally in time and $T^{*}=T$. In the case $m^{-} \geq 1$ the solution satisfies the inclusion $|v|^{m(z)-1} v_{t} \in L^{2}\left(Q_{T_{*}}\right)$.

A byproduct of the proof of Theorem 2.1 are the energy relations for the constructed strong solution. Let us introduce the energy functional

$$
E(t, v)=\int_{\Omega}\left(\sum_{i=1}^{n} a_{i} \frac{\left|D_{i} v\right|^{p_{i}}}{p_{i}}-b \frac{|v|^{\sigma}}{\sigma}\right) d x .
$$

Theorem 2.2 (Theorems 7.1, 7.2, [7]). Let the conditions of Theorem 2.1 be fulfilled. Then problem (1.1) has a strong solution which satisfies the equality: $\forall$ a.e. $t \in\left(0, T^{*}\right)$

$$
\begin{aligned}
\frac{d}{d t}\left(\int_{\Omega} \frac{m}{m+1}|v|^{m+1} d x\right)+\sum_{i=1}^{n} \int_{\Omega} a_{i}\left|D_{i} v\right|^{p_{i}} d z+\int_{\Omega} b|v|^{\sigma} d x \\
=\int_{\Omega} g v d z+\int_{\Omega} \frac{\partial_{t} m}{(m+1)^{2}}[1-(m+1) \ln |v|]|v|^{m+1} d x .
\end{aligned}
$$

Moreover, if $b(z) \geq b^{-}>0, g(z) \equiv 0$ and $0 \leq \sigma_{t}(z) \leq C_{\sigma}$ in the cylinder $Q_{T^{*}}$, then

$$
E(t, v) \leq E\left(0, v_{0}\right)+C^{*} t+\mathcal{M}(\mu) \quad \text { for a.e. } t \in\left(0, T^{*}\right),
$$

where $\mathcal{M}(s) \rightarrow 0$ as $s \rightarrow 0$ and

$$
C^{*}=|\Omega|\left(n \frac{a^{+} C_{p}}{\left(p^{-}\right)^{2}}+\left(1+\frac{1}{\mathrm{e}}\right) \frac{b^{+} C_{\sigma}}{\left(\sigma^{-}\right)^{2}}\right) .
$$


The derivation of the energy estimates (2.14), (2.15) in [7] was tightly related to the method of regularization of the degenerate equation (1.1). The sequence of solutions of the regularized problems converges to a strong solution of problem (1.1) understood in the sense of Definition 2.1, and the energy relations (2.14), (2.15) are obtained as the limits of the corresponding relations for the solutions of the regularized problems. It is not clear, however, whether the conditions on the data imposed in Theorem 2.1 are indeed essential for the validity of the energy estimates. Since the study of the localization and blow-up properties of solutions to problem (1.1) relies solely on the analysis of the energy relations (2.14), (2.15) and is practically independent of the existence theorem, it is convenient to introduce the concept of Energy Solution of problem (1.1): a function

$$
v \in C^{0}\left(0, T ; L^{m+1}(\Omega)\right) \cap \mathbf{W}\left(Q_{T}\right)
$$

is called Energy Solution of problem (1.1) if it satisfies (2.4), (2.5) and the energy relations (2.14), (2.15).

All the further considerations are confined to the class of Energy Solutions of problem (1.1), which is surely nonempty by virtue of Theorem 2.1.

\section{Sufficient conditions of the finite time blow-up} follows:

Given an energy solution $v(z)$ of problem (1.1), we define the blow-up moment $t^{*}$ as

$$
\Sigma(t) \equiv \int_{\Omega} \frac{m}{m+1}|v|^{m+1} d x \rightarrow \infty \quad \text { as } t \rightarrow t^{*}, \quad m=m(x, t) .
$$

We begin with proving several technical lemmas.

Lemma 3.1. Let $\mu^{+}$and $\mu^{-}$be positive constants, $1<\mu^{-} \leq \mu^{+}<\infty$. If a nonnegative function $f(t)$ satisfies the inequality

$$
\left\{\begin{array}{l}
\lambda f^{\prime}(t) \geq C \min \left\{f^{\mu^{+}}(t), f^{\mu^{-}}(t)\right\} \quad \text { for a.e. } t \geq 0, \\
f(0)=f_{0}>0, \quad \lambda=\text { const }>0,
\end{array}\right.
$$

then there exists a finite moment $t^{*}$ such that $f(t) \rightarrow \infty$ as $t \rightarrow t^{*}$.

Proof. Let us denote $t^{*}=\sup \{t>0: f(t)<\infty\}$. Introduce the function $w(t):=\frac{f(t)}{f_{0}}$, which satisfies the conditions

$$
\left\{\begin{array}{l}
\lambda w^{\prime}(t) \geq D \min \left\{w^{\mu^{+}}(t), w^{\mu^{-}}(t)\right\} \quad \text { for a.e. } t \in\left(0, t^{*}\right), \\
w(0)=1, \quad D=C \min \left\{f_{0}^{\mu^{+}-1}, f_{0}^{\mu^{-}-1}\right\} .
\end{array}\right.
$$

By virtue of (3.3) $w(t)$ is monotone increasing, whence $w(t)>1$ for all $t \in\left(0, t^{*}\right)$. Then (3.3) reads

$$
D w^{\mu^{-}}(t) \leq \lambda w^{\prime}(t) \quad \text { for a.e. } t \in\left(0, t^{*}\right), \quad w(0)=1,
$$


and can be directly integrated:

$$
w(t) \geq \frac{\lambda^{\frac{1}{\mu^{-}-1}}}{\left(\lambda-D\left(\mu^{-}-1\right) t\right)^{\frac{1}{\mu^{-}-1}}} .
$$

It follows that

$$
w(t) \rightarrow \infty \text { as } t \rightarrow \frac{\lambda}{D\left(\mu^{-}-1\right)}=\frac{\lambda f_{0}}{C\left(\mu^{-}-1\right) \min \left\{f_{0}^{\mu^{+}}, f_{0}^{\mu^{-}}\right\}} \equiv \theta^{*}\left(f_{0}\right)<\infty,
$$

which means that $t^{*} \leq \theta^{*}$.

Lemma 3.2. Let $\mu^{ \pm}$be positive constants, $1<\mu^{-} \leq \mu^{+}<\infty$. If a continuous function $f(t)$ satisfy the conditions

$$
\left\{\begin{array}{l}
\left(C_{1} t-C_{2}\right)+\lambda f^{\prime}(t) \geq C \min \left\{f^{\mu^{+}}(t), f^{\mu^{-}}(t)\right\} \quad \text { for a.e. } t \geq 0 \\
f(0)=f_{0}>0
\end{array}\right.
$$

with positive constants $C_{1}$ and $C_{2}$, then there exists $f_{*}>0$ such that for every initial value $f_{0}>f_{*}$ there is a finite $t^{*}$ such that $f(t) \rightarrow \infty$ as $t \rightarrow t^{*} \leq C_{1} / C_{2}$.

Proof. Let $\theta^{*}(s)$ be the monotone decreasing function defined in (3.4) and let $f_{*}$ be the solution of the equation $C_{1}\left(1+\theta^{*}\left(f_{*}\right)\right)=C_{2}$ :

$$
f_{*}= \begin{cases}\left(\frac{\lambda C_{1}}{C C_{2}\left(\mu^{-}-1\right)}\right)^{\frac{1}{\mu^{+}-1}} & \text { if } \frac{\lambda C_{1}}{C C_{2}\left(\mu^{-}-1\right)}<1, \\ \left(\frac{\lambda C_{1}}{C C_{2}\left(\mu^{-}-1\right)}\right)^{\frac{1}{\mu^{-}-1}} & \text { otherwise. }\end{cases}
$$

Under this choice of $f_{*}$ we have that $C_{1} t-C_{2}<0$ on the interval $\left(0, \theta^{*}\left(f_{0}\right)\right)$ for every $f_{0}>f_{*}$. It follows that instead of (3.5) $f(t)$ satisfies inequality (3.2) and, by virtue of Lemma 3.1,

$$
f(t) \rightarrow \infty \quad \text { as } t \rightarrow \frac{\lambda f_{0}}{C\left(\mu^{-}-1\right) \min \left\{f_{0}^{\mu^{+}}, f_{0}^{\mu^{-}}\right\}}<\theta^{*}\left(f_{*}, \mu^{-}\right) .
$$

Theorem 3.1. Let $v(z)$ be an energy solution of problem (1.1) in a cylinder $Q_{T}, T<\infty$. Assume that $g \equiv 0$ and there exist constants $b^{-}>0$ and $\delta>1$ such that $b(z) \geq b^{-}$in $Q_{T}$

$$
\sigma^{-}(t)>\delta \max \left\{m^{+}(t)+1, \max _{i=1, \ldots, n} p_{i}^{+}(t)\right\} \quad \forall(x, t) \in Q_{T} .
$$

Let $C^{*}$ and $E(t, v)$ denote the constant and the energy functional from Theorem 2.2. 
(i) If $C^{*}=0, m_{t}=0$ and $E\left(0, v_{0}\right)<0$, the solution blows-up in a finite time $t^{*}$ with

$$
t^{*} \leq \frac{1}{b^{-}\left(\sigma^{-}-p^{+}\right)\left(1-\frac{m^{+}+1}{\sigma^{-}}\right) \min \left\{\Sigma^{\mu^{+}}(0), \Sigma^{\mu^{-}}(0)\right\}}, \quad \mu^{ \pm}=\left(\frac{\sigma(z)}{m(z)+1}\right)^{ \pm} .
$$

(ii) If $C^{*}>0, m_{t}=0$ and $E\left(0, v_{0}\right)<0$, there exists $\Sigma_{*}(0)$ such that for every $\Sigma(0)>\Sigma_{*}(0)$ the solution blows-up in finite time.

(iii) If $-\mu \leq m_{t} \leq 0, C^{*}>0$ and $E\left(0, v_{0}\right)<0$,

$$
E\left(0, v_{0}\right)+\mu|\Omega|\left(1+\frac{m^{+}+1}{m^{-}+1}\right) \mathrm{e}^{\frac{m^{+}+1}{m^{-}+1}}<0,
$$

there exist $\mu^{*}>0$ and $\Sigma_{*}(0)$ such that for $0 \leq \mu<\mu^{*}$ and $\Sigma(0)>\Sigma_{*}(0)$ the solution blows-up in a finite time.

Proof. (i)-(ii) The assumption $m \equiv m(x)$ allows us to drop in (2.14), (2.15) the terms depending on $m_{t}$ and to write these relations in the form

$$
\begin{aligned}
\forall \text { a.e. } t \in(0, T) \quad \Sigma^{\prime}(t) & =\int_{\Omega}\left(-\sum_{i=1}^{n} a_{i}\left|D_{i} v\right|^{p_{i}}+b|v|^{\sigma}\right) d x, \\
E(t, v) & \leq E\left(0, v_{0}\right)+C^{*} t .
\end{aligned}
$$

Let us multiply (3.7) by a constant $\lambda>0$ and add to (3.8). Dropping the nonnegative term on the left-hand side, we obtain the inequality

$$
E(t, v)+\lambda \int_{\Omega}\left(-\sum_{i=1}^{n} a_{i}\left|D_{i} v\right|^{p_{i}}+b|v|^{\sigma}\right) d x \leq \lambda \Sigma^{\prime}(t)+\left(E\left(0, v_{0}\right)+C^{*} t\right),
$$

whence

$$
\int_{\Omega}\left(\sum_{i=1}^{n}\left(\frac{1}{p_{i}}-\lambda\right) a_{i}\left|D_{i} v\right|^{p_{i}}+b\left(\lambda-\frac{1}{\sigma}\right)|v|^{\sigma}\right) d x \leq \lambda \Sigma^{\prime}(t)+\left(E\left(0, v_{0}\right)+C^{*} t\right)
$$

Assumption (3.6) allows one to choose $\lambda$ in such a way that

$$
\frac{1}{\sigma(z)} \leq \frac{1}{\sigma^{-}(t)}<\lambda \leq \frac{1}{p^{+}(t)} \leq \frac{1}{p(z)}
$$

Plugging this inequality to (3.10) we obtain the inequality

$$
\int_{\Omega}\left(a^{-}\left(\frac{1}{p^{+}}-\lambda\right) \sum_{i=1}^{n}\left|D_{i} v\right|^{p_{i}}+b^{-}\left(\lambda-\frac{1}{\sigma^{-}}\right)|v|^{\sigma}\right) d x \leq \lambda \Sigma^{\prime}(t)+\left(E\left(0, v_{0}\right)+C^{*} t\right),
$$

in which the first term on the left-hand side is nonnegative and can be omitted: 


$$
b^{-}\left(\frac{1}{p^{+}}-\frac{1}{\sigma-}\right) \int_{\Omega}|v|^{\sigma} d x \leq \lambda \Sigma^{\prime}(t)+\left(E\left(0, v_{0}\right)+C^{*} t\right) .
$$

According to (3.6) $1+m^{+}<\sigma^{-}$in $Q_{T}$. Using the Hölder inequality (2.3) and (2.1), we have that for a.e. $t \in(0, T)$

$$
\begin{aligned}
\Sigma(t) \leq \int_{\Omega}|v|^{m+1} d x & \leq \max \left\{\|v\|_{m(\cdot, t)+1}^{m^{+}(t)+1},\|v\|_{m(\cdot, t)+1}^{m^{-}(t)+1}\right\} \\
& \leq \max \left\{\left(\int_{\Omega} v^{\sigma(z)} d x\right)^{\frac{m^{+}(t)+1}{\sigma-(t)}},\left(\int_{\Omega} v^{\sigma(z)} d x\right)^{\frac{m^{-}(t)+1}{\sigma+}(t)}\right\},
\end{aligned}
$$

whence

$$
\min \left\{\Sigma^{\frac{\sigma^{-}(t)}{m^{+}(t)+1}}(t), \Sigma^{\frac{\sigma^{+}}{m^{-}(t)+1}}(t)\right\} \leq \int_{\Omega}|v|^{\sigma(z)} d x
$$

Set

$$
\mu^{+}=\frac{\sigma^{+}}{m^{-}+1}, \quad \mu^{-}=\frac{\sigma^{-}}{m^{+}+1}, \quad C=b^{-}\left(\frac{1}{p^{+}}-\frac{1}{\sigma^{-}}\right) .
$$

Combining the last inequality with (3.11), we arrive at the differential inequality for $\Sigma(t)$ :

$$
\left\{\begin{array}{l}
C \min \left\{\Sigma^{\mu^{+}}(t), \Sigma^{\mu^{-}}(t)\right\} \leq \lambda \Sigma^{\prime}(t)+\left(E\left(0, v_{0}\right)+C^{*} t\right) \quad \text { for a.e. } t \in(0, T), \\
\Sigma(0)=\int_{\Omega} \frac{m_{0}}{m_{0}+1}\left|v_{0}\right|^{m_{0}+1} d x .
\end{array}\right.
$$

The assertions (i) and (ii) follow now from Lemmas 3.1 and 3.2.

(iii) It is assumed that $-\mu \leq m_{t} \leq 0$ a.e. in $Q_{T}$. Let us denote

$$
J(t)=\int_{\Omega} \frac{\partial_{t} m}{(m+1)^{2}}[1-(m+1) \ln |v|]|v|^{m+1} d x
$$

Noting that the integrand of $J$ is nonnegative on the set where $\ln |v|>\frac{1}{m+1}$, we estimate it as follows: $\forall t \in(0, T)$

$$
\begin{aligned}
J(t) & \geq \int_{\Omega \cap\left\{\ln |v|<\frac{1}{m+1}\right\}} \frac{\partial_{t} m}{(m+1)^{2}}[1-(m+1) \ln |v|]|v|^{m+1} d x \\
& \geq \int_{\Omega \cap\left\{\ln |v|<\frac{1}{m+1}\right\}} \frac{\partial_{t} m}{(m+1)^{2}}\left(1+\frac{m^{+}+1}{m^{-}+1}\right) \mathrm{e}^{\frac{m^{+}+1}{m^{-}+1}} d x \\
& \geq\left(1+\frac{m^{+}+1}{m^{-}+1}\right) \mathrm{e}^{\frac{m^{+}+1}{m^{-}+1}} \int_{\Omega} \frac{\partial_{t} m}{(m+1)^{2}} d x \geq-\mu L
\end{aligned}
$$


with the constant $L=|\Omega|\left(1+\frac{m^{+}+1}{m^{-}+1}\right) \mathrm{e}^{\frac{m^{+}+1}{m^{-}+1}}>1$. The energy relations (2.14), (2.15) take the form

$$
\begin{aligned}
\forall \text { a.e. } t \in(0, T) \quad & \int_{\Omega}\left(-\sum_{i=1}^{n} a_{i}\left|D_{i} v\right|^{p_{i}}+b|v|^{\sigma}\right) d x \leq \Sigma^{\prime}(t)+\mu L, \\
& E(t, v) \leq E\left(0, v_{0}\right)+C^{*} t+\mathcal{M}(\mu), \quad \mathcal{M}(\mu) \rightarrow 0 \text { as } \mu \rightarrow 0 .
\end{aligned}
$$

Gathering them and following the derivation of (3.13) we arrive at the inequality

$$
\left\{\begin{array}{l}
C \min \left\{\Sigma^{\mu^{+}}(t), \Sigma^{\mu^{-}}(t)\right\} \leq \lambda \Sigma^{\prime}(t)+\left(\lambda \mu L+\mathcal{M}(\mu)+E\left(0, v_{0}\right)\right)+C^{*} t \quad \text { in }(0, T), \\
\Sigma(0)=\int_{\Omega} \frac{m_{0}}{m_{0}+1}\left|v_{0}\right|^{m_{0}+1} d x>0 .
\end{array}\right.
$$

Since $E\left(0, v_{0}\right)<0$ and $\lambda \mu L+\mathcal{M}(\mu) \rightarrow 0$ as $\mu \rightarrow 0$, assertion (iii) follows from Lemma 3.2 .

Corollary 3.1. Theorem 3.1 states that given any negative initial energy $E\left(0, v_{0}\right)$ every energy solution blows-up in a finite time, provided that $m_{t}$ is nonpositive and appropriately small, and that $\Sigma(0)$ is sufficiently big. On the other hand, it is easy to infer from the proof of Lemmas 3.1, 3.2 that for every fixed $\mu$ and $\Sigma(0)$ one may indicate the threshold value $E_{*}<0$ such that the every energy solution blows-up if $E\left(0, v_{0}\right)<E_{*}$.

\section{Embedding inequalities in anisotropic spaces}

Let us introduce the functions

$$
\begin{aligned}
\Sigma(t) & =\int_{\Omega} \frac{m(z)}{1+m(z)}|v(z)|^{m(z)+1} d x \\
\Lambda(t) & =\sum_{i=1}^{n} \int_{\Omega}\left|D_{i} v(z)\right|^{p_{i}(z)} d x \\
\Theta(t) & =\int_{\Omega}\left(\sum_{i=1}^{n}\left|D_{i} v(z)\right|^{p_{i}(z)}+|v(z)|^{\sigma(z)}\right) d x
\end{aligned}
$$

and denote

$$
\frac{1}{p^{ \pm}(t)}=\frac{1}{n} \sum_{i=1}^{n} \frac{1}{p_{i}^{ \pm}(t)} .
$$

We will rely on the following known result. 
Lemma 4.1 ([10, 14, 16]). Let $\Omega \subset \mathbb{R}^{n}$ be a bounded domain with Lipschitz-continuous boundary. Denote $W_{0}^{1, \vec{p}}(\Omega) \equiv\left\{u \in W_{0}^{1,1}(\Omega): D_{i} u \in L^{p_{i}}(\Omega), i=\overline{1, n}\right\}, p_{i}>1$. Let

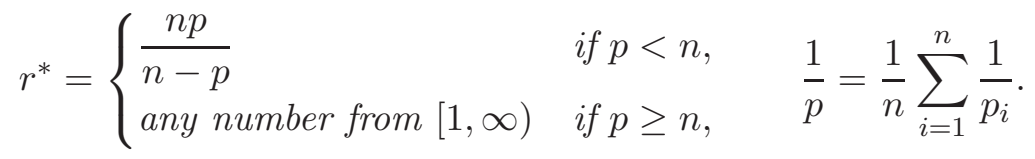

For $r \in\left[1, r^{*}\right]$ the embedding $W_{0}^{1, \vec{p}}(\Omega) \hookrightarrow L^{r}(\Omega)$ is continuous and for every $u \in W_{0}^{1, \vec{p}}(\Omega)$

$$
\|u\|_{r, \Omega} \leq C(\Omega, n)\left(\prod_{i=1}^{n}\left\|D_{i} u\right\|_{p_{i}, \Omega}\right)^{\frac{1}{n}}
$$

with an independent of $u$ constant $C$. For $r \in\left[1, r^{*}\right)$ the embedding is compact.

For any given positive constant $L$ we denote

$$
\mathcal{B}_{L}=\left\{v \in \mathbf{W}\left(Q_{T}\right): \sup _{(0, T)} \Sigma(t)+\int_{0}^{T} \Lambda(t) d t \leq L\right\} .
$$

Lemma 4.2. Let the exponents $m(z), p_{i}(z)$ satisfy conditions (2.6). If

$$
m^{+}(t)+1 \leq \begin{cases}\frac{n p^{-}(t)}{n-p^{-}(t)} & \text { if } p^{-}(t)<n, \\ \text { any number from }[1, \infty) & \text { if } p^{-}(t) \geq n,\end{cases}
$$

then for every $v \in \mathcal{B}_{L}$ the energy functions $\Sigma(t), \Lambda(t)$ satisfy the inequality

$$
\forall \text { a.e. } t \in(0, T) \quad \Sigma^{\nu^{+}(t)}(t) \leq C \Lambda(t) \quad \text { with } \frac{1}{\nu^{+}(t)}=\frac{m^{-}(t)+1}{m^{+}(t)} \frac{1}{p^{+}(t)}
$$

with $p^{+}(t)$ defined in (4.1) and a constant $C$ depending on the constants in $(2.6), n,|\Omega|$ and $L$, but independent of $v$.

Proof. Given $u \in \mathcal{B}_{L}$, let us introduce the function $v=\gamma u$ with a constant $\gamma \in(0,1]$. Denote by $\Sigma_{v}(t)$ and $\Lambda_{v}(t)$ be the energy functions associated with $v$. It is easy to calculate that

$$
\Sigma_{v}(t)+\int_{0}^{T} \Lambda_{v}(t) d t \leq\left(\gamma^{m^{-}+1}+\max _{i} \gamma^{p_{i}^{-}}\right)\left(\Sigma(t)+\int_{0}^{T} \Lambda(t) d t\right) \leq 2 \gamma L,
$$

whence $v \in \mathcal{B}_{2 \gamma L}$. If we choose $\gamma$ from the equality $2 \gamma L\left(1+m^{+}\right)=m^{-}$, then

$$
v \in \mathcal{B}_{1}, \quad \sup _{t \in(0, T)}\|v\|_{m(\cdot, t)+1, \Omega} \leq 1
$$

Let us consider first the case when $v$ satisfies conditions (4.3). According to (2.2)

$$
\Sigma(t) \leq \frac{m^{+}}{m^{-}+1} \max \left\{\|v(\cdot, t)\|_{m+1, \Omega}^{m^{+} 1},\|v(\cdot, t)\|_{m+1, \Omega}^{m^{-}+1}\right\} \leq \frac{m^{+}}{m^{-}+1}\|v(\cdot, t)\|_{m+1, \Omega}^{m^{-}+1} .
$$


By Lemma 4.1 for every $t \in[0, T]$

$$
\begin{aligned}
\|v(\cdot, t)\|_{m+1, \Omega} & \leq C\|v(\cdot, t)\|_{m^{+}(t)+1, \Omega} \\
& \leq C\left(\prod_{i=1}^{n}\left\|D_{i} v(\cdot, t)\right\|_{p_{i}^{-}(t), \Omega}\right)^{\frac{1}{n}} \leq C\left(\prod_{i=1}^{n}\left\|D_{i} v(\cdot, t)\right\|_{p_{i}(\cdot, t), \Omega}\right)^{\frac{1}{n}} .
\end{aligned}
$$

The right-hand side of this inequality can be estimated by means of (2.2): for every fixed $t \in[0, T]$

$$
\begin{aligned}
\left\|D_{i} v\right\|_{p_{i}, \Omega} & \leq \max \left\{\left(\int_{\Omega}\left|D_{i} v\right|^{p_{i}} d x\right)^{\frac{1}{p_{i}^{-}(t)}},\left(\int_{\Omega}\left|D_{i} v\right|^{p_{i}} d x\right)^{\frac{1}{p_{i}^{+}(t)}}\right\} \\
& \leq \max \left\{\Lambda^{\frac{1}{p_{i}^{-}(t)}}(t), \Lambda^{\frac{1}{p_{i}^{+}(t)}}(t)\right\}
\end{aligned}
$$

whence

$$
\begin{aligned}
&\|v(\cdot, t)\|_{m+1, \Omega} \leq C\left(\prod_{i=1}^{n} \max \left\{\Lambda^{\frac{1}{p_{i}^{-}(t)}}(t), \Lambda^{\frac{1}{p_{i}^{+}(t)}}(t)\right\}\right)^{\frac{1}{n}} \\
& \leq C\left\{\begin{array} { l l } 
{ ( \prod _ { i = 1 } ^ { n } \Lambda ^ { \frac { 1 } { p _ { i } ^ { + } ( t ) } } ( t ) ) ^ { \frac { 1 } { n } } } & { \text { if } \Lambda ( t ) \leq 1 , } \\
{ ( \prod _ { i = 1 } ^ { n } \Lambda ^ { \frac { 1 } { p _ { i } ^ { - } ( t ) } } ( t ) ) ^ { \frac { 1 } { n } } } & { \text { if } \Lambda ( t ) > 1 , }
\end{array} \equiv C \left\{\begin{array}{ll}
\Lambda^{\frac{1}{p^{+}(t)}}(t) & \text { if } \Lambda(t) \leq 1, \\
\Lambda^{\frac{1}{p^{-}(t)}}(t) & \text { if } \Lambda(t)>1 .
\end{array}\right.\right.
\end{aligned}
$$

Using (4.4) and (2.2) we obtain the inequality

$$
\left(\frac{1+m^{-}}{m^{+}} \Sigma(t)\right)^{\frac{p^{+}(t)}{1+m^{-}(t)}} \leq\|v(\cdot, t)\|_{m+1, \Omega}^{p^{+}(t)}=\min \left\{\|v(\cdot, t)\|_{m+1, \Omega}^{p^{-}(t)},\|v\|_{m+1, \Omega}^{p^{+}(t)}\right\} \leq C \Lambda(t),
$$

which yields (4.2), provided that $v(z)$ satisfies (4.3). In the general case (4.2) holds for the function $w=\gamma v$ with $\gamma=m^{-} /\left(2 L\left(1+m^{+}\right)\right)$, which gives estimate (4.2) for $v$.

Let us accept the notation

$$
\mathcal{G}_{L}=\left\{v \in \mathbf{W}\left(Q_{T}\right) \cap L^{\sigma}\left(Q_{T}\right): \sup _{(0, T)} \Sigma(t)+\int_{0}^{T} \Theta(t) d t \leq L\right\} .
$$

Lemma 4.3. Let the exponents $m, p_{i}, \sigma$ satisfy conditions (2.6). If

$$
\left\{\begin{array}{l}
\sigma^{-}+m^{-}>2 \\
\frac{m^{+}(t) \sigma^{+}(t)}{\sigma^{-}(t)-1} \leq \frac{n p^{-}(t)}{n-p^{-}(t)} \quad \text { if } p^{-}(t)<n
\end{array}\right.
$$

then for every $u \in \mathcal{G}_{L}$ 


$$
\forall \text { a.e. } t \in(0, T) \quad \Sigma(t) \leq C \Theta^{\frac{1}{\nu^{+}(t)}}(t), \quad \frac{1}{\nu^{+}(t)}=\frac{1}{\sigma^{+}(t)}+\frac{m^{-}(t)}{p^{+}(t)}
$$

with $p^{+}(t)$ defined in (4.1) and a constant $C$ depending on $n,|\Omega|$, the constants in (2.6) and $L$, but independent of $u$.

Proof. The proof imitates the proof of the previous lemma. It is sufficient to prove the assertion for the functions $u \in \mathbf{W}\left(Q_{T}\right) \cap L^{\sigma}\left(Q_{T}\right)$ satisfying the inequality

$$
\sup _{t \in(0, T)}\|u\|_{m(\cdot, t)+1, \Omega} \leq 1 .
$$

Observe first that by virtue of Lebesgue's differentiation theorem $\Theta(t)$ is well-defined for a.a. $t \in(0, T)$ and every $u \in \mathcal{G}_{L}$. Following the proof of Lemma 4.2 we find that under assumption (4.5) for a.e. $t \in(0, T)$

$$
\left\||u(\cdot, t)|^{m(\cdot, t)}\right\|_{\frac{\sigma(\cdot, t)}{\sigma(\cdot, t)-1}, \Omega} \leq C\|u(\cdot, t)\|_{\frac{m^{+}(t) \sigma+(t)}{\sigma^{-}(t)-1}, \Omega}^{m^{-}(t)} \leq C \begin{cases}\Lambda^{\frac{m^{-}(t)}{p^{+}(t)}}(t) & \text { if } \Lambda(t) \leq 1, \\ \Lambda^{\frac{m^{-}(t)}{p^{-}(t)}}(t) & \text { if } \Lambda(t)>1 .\end{cases}
$$

Combining this inequality with Hölder's inequality (2.3) we obtain the estimate

$$
\begin{aligned}
& \int_{\Omega}|u(x, t)|^{m+1} d x \leq\left\||u(\cdot, t)|^{m(\cdot, t)}\right\|_{\frac{\sigma(\cdot, t)}{\sigma(\cdot, t)}, \Omega}\|u(\cdot, t)\|_{\sigma(\cdot, t), \Omega} \\
& \leq C \max \left\{\left(\int_{\Omega}|u|^{\sigma(z)} d x\right)^{\frac{1}{\sigma^{+}(t)}},\left(\int_{\Omega}|u|^{\sigma(z)} d x\right)^{\frac{1}{\sigma^{-}(t)}}\right\} \\
& \times \max \left\{\Lambda^{\frac{m^{-}(t)}{p^{+}(t)}}(t), \Lambda^{\frac{m^{-}(t)}{p^{-}(t)}}(t)\right\} \leq C \begin{cases}\Theta^{\frac{1}{\nu^{+}(t)}}(t) & \text { if } \Theta(t)<1, \\
\Theta^{\frac{1}{\nu^{-}(t)}}(t) & \text { if } \Theta(t) \geq 1 .\end{cases}
\end{aligned}
$$

Using assumption (4.7) we arrive at the inequality

$$
\begin{aligned}
& \left(\frac{1+m^{-}}{m^{+}} \Sigma(t)\right)^{\nu^{+}(t)} \leq\left(\int_{\Omega}|u(z)|^{m+1} d x\right)^{\nu^{+}(t)} \\
& \quad \leq \min \left\{\left(\int_{\Omega}|u(z)|^{m+1} d x\right)^{\nu^{+}(t)},\left(\int_{\Omega}|u(z)|^{m+1} d x\right)^{\nu^{-}(t)}\right\} \leq C \Theta(t) .
\end{aligned}
$$

The general case reduces to the considered one by means of scaling $v=\gamma u$ with a constant $\gamma \in(0,1]$ such that

$$
\begin{aligned}
& \Sigma_{v}(t)+\int_{0}^{T} \Theta_{v}(t) d t \leq\left(\gamma^{m^{-}+1}+\max _{i} \gamma^{p_{i}^{-}}+\gamma^{\sigma^{-}}\right)\left(\Sigma(t)+\int_{0}^{T} \Theta(t) d t\right) \leq 3 \gamma L, \\
& \int_{\Omega}|v|^{m+1} d x \leq \frac{1+m^{+}}{m^{-}} \Sigma_{v}(t) \leq 3 \frac{1+m^{+}}{m^{-}} \gamma L \leq 1 .
\end{aligned}
$$




\section{Ordinary differential inequalities for the energy function}

Let $u \in \mathbf{W}\left(Q_{T}\right)$ be an energy solution of problem (1.1). We assume that

$$
g(z) \in L^{\infty}\left(0, T ; L^{1}(\Omega)\right), \quad b(z) \leq 0 \text { and } 0 \leq m_{t} \leq \mu \text { a.e. in } Q_{T} .
$$

Under these assumptions (2.14) takes on the form: $\forall$ a.e. $t \in(0, T)$

$$
\begin{aligned}
\Sigma^{\prime}(t) & +\int_{\Omega}\left(a^{-} \sum_{i=1}^{n}\left|D_{i} u(z)\right|^{p_{i}(z)}-b(z)|u(z)|^{\sigma(z)}\right) d x \\
& \leq \int_{\Omega} u g(z) d x+\int_{\Omega} \frac{m_{t}}{(m+1)^{2}}(1-(m+1) \ln |u|)|u|^{m+1} d x .
\end{aligned}
$$

Moreover, we will assume that the solution is bounded for all $t \in(0, T)$ (cf. with Theorem 2.1): there exists a constant $l<\infty$ such that

$$
\operatorname{ess} \sup _{(0, T)}\|u(\cdot, t)\|_{\infty, \Omega} \leq l .
$$

This assumption yields boundedness of the energy function $\Sigma(t)$ :

$$
\Sigma(t)=\int_{\Omega}|u|^{m+1} d x \leq|\Omega| \max \left\{l^{m^{+}+1}, l^{m^{-}+1}\right\} \equiv q .
$$

Let us observe that

$$
\begin{aligned}
\int_{\Omega} & \frac{m_{t}}{(m+1)^{2}}(1-(m+1) \ln |u|)|u|^{m+1} \\
\quad & \int_{\Omega} \frac{m_{t}}{(m+1)^{2}}|u|^{m+1} d x-\int_{\Omega} \frac{m_{t}}{m+1} \ln |u||u|^{m+1} d x \\
& \leq \operatorname{ess} \sup _{\Omega}\left(\frac{m_{t}}{(m+1)^{2}}\right) \Sigma(t)-\operatorname{ess} \sup _{\Omega}\left(\frac{m_{t}}{m+1}\right) \int_{\Omega \cap\{|u|<1\}}|u|^{m+1} \ln |u| d x \\
& \equiv K_{1}(t) \Sigma(t)+K_{2}(t) I(t) .
\end{aligned}
$$

Fix $t \in(0, T)$, some $\alpha \in\left(0, m^{-}(t)+1\right)$ and represent the integral $I(t)$ in the form

$$
I(t)=\int_{\Omega \cap\{|u|<1\}}|u|^{m+1-\alpha}\left(|u|^{\alpha}|\ln | u||\right) d x .
$$

It is easy to see that the function $s^{\alpha}|\ln s|$ with $\alpha \in\left(0, m^{-}(t)+1\right)$, defined on the interval $(0,1)$, vanishes as $s \rightarrow 0, s \rightarrow 1$ and attains the maximum value at the point $s=\mathrm{e}^{-\frac{1}{\alpha}}$ :

$$
\max _{[0,1]}\left(s^{\alpha}|\ln s|\right)=\frac{1}{\alpha \mathrm{e}} .
$$

Using this fact, applying Hölder's inequality (2.3) and then (2.2), we estimate 


$$
\begin{aligned}
I(t) & \leq \frac{1}{\alpha \mathrm{e}} \int_{\Omega \cap\{|u|<1\}}|u|^{m+1-\alpha} d x \leq \frac{1}{\alpha \mathrm{e}} \int_{\Omega}|u|^{m+1-\alpha} d x \\
& \leq \frac{2}{\alpha \mathrm{e}}\|1\|_{\frac{m(\cdot, t)+1}{\alpha}, \Omega}\left\||u|^{m+1-\alpha}\right\|_{\frac{m(\cdot, t)+1}{m(\cdot, t)+1-\alpha}, \Omega} \\
& \leq \frac{2}{\alpha \mathrm{e}}\|1\|_{\frac{m(\cdot, t)+1}{\alpha}, \Omega} \max \left\{\Sigma^{1-\frac{\alpha}{m^{+}(t)+1}}(t), \Sigma^{1-\frac{\alpha}{m^{-}(t)+1}}(t)\right\} .
\end{aligned}
$$

Recall that according to the definition of the Luxemburg norm

$$
\frac{2}{\alpha \mathrm{e}}\|1\|_{\frac{m(\cdot, t)+1}{\alpha}, \Omega} \leq \frac{2}{\alpha \mathrm{e}} \max \left\{|\Omega|^{\frac{\alpha}{m^{+}(t)+1}},|\Omega|^{\frac{\alpha}{m^{-}(t)+1}}\right\} \equiv \lambda .
$$

Applying Lemmas 4.2, 4.3 to the left-hand side of (5.2), we continue it in the following way: $\forall$ a.e. $t \in(0, T)$

$$
\begin{aligned}
\Sigma^{\prime}(t)+C \Sigma^{\nu^{+}(t)}(t) & \leq l \int_{\Omega}|g(z)| d x+K_{1}(t) \Sigma(t) \\
& +\lambda K_{2}(t) \max \left\{\Sigma^{1-\frac{\alpha}{m^{+}(t)+1}}(t), \Sigma^{1-\frac{\alpha}{m^{-}(t)+1}}(t)\right\},
\end{aligned}
$$

where $\nu^{+}(t)$ is the variable exponent defined by formula (4.2) if $b^{+}=0$, and by formula (4.6) if $b^{+}<0$. Let

$$
M=\max \{1, q\}, \quad Z(t)=\frac{\Sigma(t)}{M} \leq 1 .
$$

Dividing (5.4) by $M$ we arrive at the inequality for $Z(t)$

$$
\left\{\begin{aligned}
& Z^{\prime}(t)+C M^{\nu^{+}(t)-1} Z^{\nu^{+}(t)}(t) \leq \frac{l}{M} \int_{\Omega} g(x, t) d x \\
&+ K_{1}(t) Z(t)+\lambda K_{2}(t) M^{-\frac{\alpha}{m^{+}(t)+1}} Z^{1-\frac{\alpha}{m^{-}(t)+1}}(t) \\
& C=\text { const } \text { is defined in (4.2) if } b^{+}=0, \text { or in (4.6) if } b^{+}<0 .
\end{aligned}\right.
$$

Finally, multiplying (5.6) by the function $\mathrm{e}^{-K(t)}$ with

$$
K(t) \equiv \int_{0}^{t} K_{1}(\tau) d \tau=\int_{0}^{t} \operatorname{ess} \sup _{\Omega}\left(\frac{m_{t}}{(m+1)^{2}}\right) d \tau,
$$

we obtain the differential inequality for $S(t) \equiv Z(t) \mathrm{e}^{-K(t)}: \forall$ a.e. $t \in(0, T)$

$$
S^{\prime}(t)+D(t) S^{\nu^{+}(t)}(t) \leq \phi(t)+\rho(t) S^{1-\frac{\alpha}{m^{-}(t)+1}}(t)
$$

with the right-hand side

$$
\phi(t)=\frac{l \mathrm{e}^{-K(t)}}{M} \int_{\Omega} g(z) d x
$$

and the coefficients 


$$
\begin{aligned}
& D(t)=C(\mathrm{e} M)^{-K(t)\left(1-\nu^{+}(t)\right)} \\
& \rho(t)=\lambda K_{2}(t) M^{-\frac{\alpha}{m^{+}(t)+1}} \exp \left(K(t)\left(\left(1-\frac{\alpha}{m^{-}(t)+1}\right)\right) .\right.
\end{aligned}
$$

The derivation of inequality (5.8) remains true if the exponent $\alpha$ depends on $t$. Let us choose $\alpha \equiv \alpha(t) \in\left(0, m^{-}(t)+1\right)$ in the special way:

$$
\nu^{+}(t)=1-\frac{\alpha(t)}{m^{-}(t)+1} \quad \Longleftrightarrow \quad \alpha(t)=\left(m^{-}(t)+1\right)\left(1-\nu^{+}(t)\right) .
$$

Then the second term on the right-hand side right of (5.8) transforms into $\rho(t) S^{\nu^{+}(t)}(t)$ and (5.8) leads to the inequality

$$
\forall \text { a.e. } t \in(0, T) \quad S^{\prime}(t)+(D(t)-\rho(t)) S^{\nu^{+}(t)}(t) \leq \phi(t) .
$$

The above arguments are summarized in the following lemma.

Lemma 5.1. Let $u \in \mathbf{W}\left(Q_{T}\right)$ be a bounded energy solution of problem (1.1). Assume that the conditions of Lemma 4.2 or Lemma 4.3, and conditions (5.1)-(5.2) are fulfilled. Then the function

$$
S(t)=\frac{\mathrm{e}^{-K(t)}}{M} \int_{\Omega}|u|^{m(x, t)+1} d x
$$

satisfies the differential inequality (5.11) with $K(t), D(t), \rho(t)$ and $M$ defined in (5.7), (5.10) and (5.5).

Lemma 5.2. Let the conditions of Lemma 5.1 be fulfilled. Assume that in condition (5.3) $q \leq 1$. If the functions $m(z), m_{t}(z)$ satisfy the inequality

$$
4 \frac{m_{t}^{+}(t)}{\alpha(t) \mathrm{e}} \mathrm{e}^{K(t)} \max \{1,|\Omega|\} \leq C \quad \forall t \in(0, T)
$$

with the constant $C$ from (5.6), then the energy function $S(t)$ satisfies the differential inequality

$$
\forall \text { a.e. } t \in(0, T) \quad S^{\prime}(t)+\frac{1}{2} D(t) S^{\nu^{+}(t)}(t) \leq \phi(t), \quad D(t)=C \mathrm{e}^{-K(t)\left(1-\nu^{+}(t)\right)},
$$

with the right-hand side $\phi(t)$ defined in (5.9).

Proof. It suffices to check that

$$
\rho(t) \leq \frac{1}{2} D(t) \quad \Longleftrightarrow \quad 2 \lambda K_{2}(t) \leq C \mathrm{e}^{-K(t)} M^{-K(t) \frac{\alpha(t)}{m^{-}(t)+1}+\frac{\alpha(t)}{m^{+}(t)+1}} .
$$

In the conditions of the lemma $q \leq 1$ and $M=1$, so that the required inequality follows from the inequality 


$$
2 \lambda K_{2}(t) \leq C \mathrm{e}^{-K(t)}
$$

Since

$$
\frac{\alpha(t)}{m^{ \pm}(t)+1}=\frac{m^{-}(t)+1}{m^{ \pm}(t)+1}\left(1-\nu^{+}(t)\right) \quad \Longrightarrow \quad|\Omega|^{\frac{\alpha(t)}{m^{ \pm}(t)+1}} \leq \max \{1,|\Omega|\}
$$

the last inequality if fulfilled by virtue of (5.12).

Remark 5.1. Assumption (5.3) is merely technical and can be relaxed. Nonetheless, we prefer to keep it for the sake of simplicity of presentation.

Remark 5.2. 1. Condition (5.12) is easy to satisfy if $m$ is independent of $x$. In this case

$$
K(t)=\int_{0}^{t} \frac{m_{t}}{(m+1)^{2}} d \tau=\frac{1}{m(0)+1}-\frac{1}{m(t)+1}<\frac{1}{m(0)+1}<1
$$

and (5.12) becomes

$$
\frac{4 m^{\prime}(t)}{(m(t)+1)\left(1-\nu^{+}(t)\right)} \operatorname{emax}\{1,|\Omega|\} \leq C
$$

If $b^{+}=0$, the last inequality is equivalent to the inequality

$$
\frac{4 m^{\prime}(t)}{m(t)\left(1-p^{+}(t)\right)+1} \text { e } \max \{1,|\Omega|\} \leq C,
$$

which relates the admissible growth of $m(t)$ to the behavior of $p(x, t)$.

2. It is to be specially noted that inequality (5.13) remains true even when $\nu^{+}(t) \rightarrow 1$ as $t \rightarrow \infty$, i.e., when $\alpha(t) \rightarrow 0$. In the case $m \equiv m(t), b^{+}=0$ the admissible behavior of the exponents $m(t)$ and $p(x, t)$ is defined by inequality (5.14).

\section{Extinction in a finite time}

\subsection{The regular case}

Inequality (5.13) provides the analytical background for the study of the localization properties of solutions to problem (1.1). In the study of this inequality we follow the ideas of the paper [6].

Theorem 6.1 (Vanishing in a finite time). Let $v(z)$ be a bounded energy solution of problem (1.1). Assume that $v$ satisfies the energy estimate (2.14) and that either the conditions of Lemma 4.2, or the conditions of Lemma 4.3 are fulfilled. Let $\nu^{+}(t)$ denotes the exponent defined in (4.2) if $b^{+}=0$, or by (4.6) if $b^{+}<0$. If

(a) the conditions of Lemma 5.2 are fulfilled,

(b) $1>\nu_{0} \geq \nu^{+}(t)$ and $\frac{1}{n}+\frac{1}{m^{+}(t)+1} \geq \frac{1}{p^{-}(t)}$ if $n>p^{-}(t)$, 
(c) $g \equiv 0$ a.e. in $Q_{T}$,

(d) in the case $K(t) \not \equiv 0$, the initial value $\Sigma(0)$ satisfies the inequality

$$
\Sigma^{1-\nu_{0}}(0)<\frac{1}{2} C\left(1-\nu_{0}\right) \int_{0}^{\infty} \mathrm{e}^{-K(\tau)\left(1-\nu^{+}(\tau)\right)} d \tau
$$

with $K(t)$ from (5.7), and the constant $C$ from (5.6),

then there exists a finite $t_{*}$ such that $\|v\|_{m+1, \Omega}(t) \equiv 0$ for all $t \geq t_{*}$.

Proof. The assertion of the theorem will follow if we prove that $S(t) \equiv 0$ for all $t$ from some $t_{*}$ on. Under the conditions of the theorem the energy function

$$
S(t)=\mathrm{e}^{-K(t)} \int_{\Omega} \frac{m}{m+1}|u|^{m+1} d x
$$

satisfies the differential inequality (5.13) with $\phi \equiv 0$. Let us define

$$
t_{*}=\inf \left\{t>0: \forall \theta \in\left[0, t_{*}\right) S(\theta)>0\right\} .
$$

A straightforward integration of (5.13) over the interval $(0, t)$ with $t \leq t_{*}$ yields the relation (in the case $K \neq 0$ )

$$
0 \leq S^{1-\nu_{0}}(t) \leq \Sigma^{1-\nu_{0}}(0)-\frac{1}{2} C\left(1-\nu_{0}\right) \int_{0}^{t} \mathrm{e}^{-K(\tau)\left(1-\nu^{+}(\tau)\right)} d \tau \equiv \mathcal{R}(t)
$$

$\mathcal{R}(t)$ is a continuous function of $t, \mathcal{R}(0)>0$ by the definition and, due to $(6.1), \mathcal{R}(t)<0$ for the sufficiently big $t$. It follows from that there is a finite $t_{*}$ such that $\mathcal{R}\left(t_{*}\right)=0$ and, respectively, $\Sigma\left(t_{*}\right)=0$. Since $\Sigma(t)$ is nonnegative and decreasing, it is necessary that $\Sigma(t) \equiv 0$ for all $t \geq t_{*}$. In the case $K=0$ integration of (5.13) leads to the inequality

$$
0 \leq \Sigma^{1-\nu_{0}}(t) \leq \Sigma^{1-\nu_{0}}(0)-C\left(1-\nu_{0}\right) t
$$

and the conclusion follows in the same way with $t_{*}=\Sigma^{1-\nu_{0}}(0) /\left(C\left(1-\nu_{0}\right)\right)$.

Theorem 6.2 (Vanishing at a prescribed moment). Let the conditions of Theorem 6.1 be fulfilled and $g(z) \equiv 0$ for a.e. $t \geq t_{g}$. Set

$$
R^{1-\nu_{0}}(t)=\frac{1}{2} C\left(1-\nu_{0}\right) \int_{t}^{t_{g}} \mathrm{e}^{1-\nu^{+}(\tau)} d \tau
$$

If the integral

$$
\mathcal{N}\left(t_{g}\right)=\frac{1}{M} \int_{0}^{t_{g}} \frac{\phi(\tau)}{R(\tau)} d \tau
$$

is convergent and

$$
\Sigma(0)-R(0)\left(M-\mathcal{N}\left(t_{g}\right)\right) \leq 0
$$


then

$$
\|v(\cdot, t)\|_{m+1, \Omega} \equiv 0 \text { in } \Omega \text { for all } t \geq t_{g} .
$$

Proof. The energy function $S(t)=\Sigma(t) \mathrm{e}^{-K(t)}$ satisfies the nonlinear differential inequality (5.13) with the nonzero right-hand side $\phi(t)$. The proof is based on comparison of $S(t)$ with the solution of the homogeneous differential equation

$$
R^{\prime}(t)+\frac{1}{2} D(t) R^{\nu_{0}}(t)=0, \quad R(0)=R_{0}
$$

with the initial value chosen according to the condition

$$
R_{0}^{1-\nu_{0}}=\frac{1}{2} C\left(1-\nu_{0}\right) \int_{t}^{t_{g}} \mathrm{e}^{1-\nu^{+}(\tau)} d \tau
$$

The details of the proof can be found in [6, Theorem 7.1].

\subsection{The limit case}

The differential inequality (5.13) remains true if $\nu^{+}(t) \rightarrow 1$ as $t \rightarrow \infty$, but the proof of Theorem 6.1 is no longer valid because $\nu^{+}(t)$ is not separated away from the critical value. Nonetheless, under certain assumptions on $\nu^{+}(t)$ the solutions of problem (1.1) still may display the property of vanishing in a finite time. We will need the following technical lemma.

Lemma 6.1. Let a nonnegative function $S(t)$ satisfy the conditions

$$
\left\{\begin{array}{c}
S^{\prime}(t)+\frac{1}{2} C \mathrm{e}^{-K(t)\left(1-\nu^{+}(t)\right)} S^{\nu^{+}(t)}(t) \leq 0 \text { for a.e. } t \geq 0 \\
\text { with } \nu^{+}(t) \in(0,1) \text { and } C=\text { const }>0 \\
0<S(t) \leq S(0)<\infty,
\end{array}\right.
$$

$K(t)$ is a nonnegative function, $K(0)=0$. If the exponent $\nu^{+}(t)$ is monotone increasing, then $S(t) \equiv 0$ for all $t \geq t_{*}$ with $t_{*}$ defined from the equality

$$
C \int_{0}^{t_{*}} \frac{S^{\nu^{+}(s)-1}(0)}{\mathrm{e}^{K(s)\left(1-\nu^{+}(s)\right)}} d s=2 \int_{0}^{\infty} \frac{d z}{\mathrm{e}^{z\left(1-\nu^{+}(z)\right)}} .
$$

Proof. The proof is an adaptation of the proof of [6, Lemma 9.1]. Let us consider the function $J(t)=S(t) / S(0)$ which satisfying the conditions

$$
\forall \text { a.e. } t>0 \quad J^{\prime}(t)+C \mathrm{e}^{-K(t)\left(1-\nu^{+}(t)\right)} S^{\nu^{+}(t)-1}(0) J^{\nu^{+}(t)} \leq 0, \quad J(0)=1, \quad J^{\prime}(t) \leq 0 .
$$

Introducing the new independent variable

$$
\tau=C \int_{0}^{t}\left(S(0) \mathrm{e}^{K(\theta)}\right)^{\nu^{+}(\theta)-1} d \theta
$$

the new exponent $a(\tau) \equiv \nu^{+}(t)$ and the new thought function $I(\tau) \equiv J(t)$, we find that 


$$
\begin{array}{ll}
\forall \text { a.e. } \tau>0 \quad & I^{\prime}(\tau)+I^{a(\tau)} \leq 0, \\
& I(0)=1, \quad I(\tau) \geq 0, \quad I^{\prime}(\tau) \leq 0 .
\end{array}
$$

By monotonicity of $I(\tau)$ there is an interval $[0, \epsilon)$ where $I(\tau)>0$, otherwise $I(\tau) \equiv 0$ for all $\tau \geq 0$. Since $I(\tau) \leq 1$ and $a(\tau) \in(0,1)$, then $I(\tau) \leq I^{a(\tau)}(\tau)$, which leads to the inequality

$$
I^{\prime}(\tau)+I(\tau) \leq I^{\prime}(\tau)+I^{a(\tau)}(\tau) \leq 0 .
$$

This inequality yields $I(\tau) \leq e^{-\tau}$, whence

$$
\tau \leq-\ln I(\tau) \quad \text { and } \quad a(\tau) \leq a(-\ln I(\tau)) .
$$

Combining this inequality with (6.3) we have that

$$
I^{\prime}(\tau)+I^{a(-\ln I(\tau))}(\tau) \leq 0
$$

The straightforward integration of this inequality over the interval $(0, \tau)$ gives:

$$
\int_{1}^{I(\tau)} \frac{d r}{r^{a(-\ln r)}} \leq-\tau
$$

Introducing the new variable $z=-\ln r$, we rewrite it in the form

$$
\int_{0}^{-\ln I(\tau)} \frac{d z}{\mathrm{e}^{z(1-a(z))}} \geq \tau \quad \text { for } \tau \geq 0 .
$$

According to the choice of $t_{*}$

$$
\int_{0}^{-\ln I\left(\tau_{*}\right)} \frac{d z}{\mathrm{e}^{z(1-a(z))}} \geq \tau_{*}=\int_{0}^{\infty} \frac{d z}{\mathrm{e}^{z(1-a(z))}},
$$

which is impossible unless $I\left(\tau_{*}\right)=S\left(t_{*}\right) / S(0)=0$.

Theorem 6.3 (Extinction of solutions of eventually linear equations). Let $v(z)$ be an energy solution of problem (1.1) with $\Sigma_{0}=\int_{\Omega}\left|v_{0}\right|^{m(x, 0)+1} d x<\infty$ and $g \equiv 0$. Assume that $|v| \leq l$ a.e. in $Q_{T}$ for some $l=$ const. $\geq 1, m_{t} \geq 0$ a.e. in $Q_{T}$, and that one of the following conditions holds:

- $b(z) \leq 0, \quad \nu^{+}(t)=\frac{m^{+}(t) p^{+}(t)}{1+m^{-}(t)} \rightarrow 1 \quad$ as $t \rightarrow \infty, \quad \nu^{+}(t)$ is monotone increasing,

- $b(z)<b^{+}<0, \quad \nu^{+}(t)=\frac{\left(\sigma^{+}(t)+m^{+}(t)-1\right) p^{+}(t)}{\sigma^{+}(t)+m^{+}(t)} \rightarrow 1 \quad$ as $t \rightarrow \infty, \quad \nu^{+}(t)$ is monotone increasing. 


$$
\int_{0}^{\infty}\left(\Sigma_{0} \mathrm{e}^{K(s)}\right)^{\nu^{+}(s)-1} d s=\infty, \quad \int_{0}^{\infty} \frac{d s}{\mathrm{e}^{s\left(1-\nu^{+}(s)\right)}}<\infty
$$

with $K(t)$ defined in (5.7), then there exists $t_{*} \in(0, \infty)$ such that $v(x, t) \equiv 0$ in $\Omega$ for all $t \geq t_{*}$, provided that $\mu$ and $\Sigma_{0}$ are sufficiently small.

Proof. Since the energy function satisfies the differential inequality (5.13), the assertion immediately follows from Lemma 6.1.

\subsection{Equations with critical growth}

Let $v(z) \in C^{0}\left(0, T ; L^{m(x, t)+1}(\Omega)\right) \cap \mathbf{W}\left(Q_{T}\right)$ be an energy solution of the problem

$$
\left\{\begin{array}{l}
\frac{d}{d t}\left(|v|^{m(z)-1} v\right)=\sum_{i=1}^{n} D_{i}\left(a_{i}(z)\left|D_{i} v\right|^{p_{i}(z)-2} D_{i} v\right) \\
\quad+c(z)|v|^{m(z)-1} v+b(z)|v|^{\sigma(z)-2} v+g \text { in } Q_{T}, \\
v=0 \text { on } \Gamma_{T}, \\
v(x, 0)=v_{0}(x) \text { in } \Omega .
\end{array}\right.
$$

Assume that that the data of the problem satisfy the foregoing assumptions and that $c(z) \leq$ $\gamma(t)$ a.e. in $Q_{T}$ with a bounded function $\gamma(t)$. Taking $v$ for the test-function, we arrive at inequality (5.4) with the coefficient $K_{1}(t)$ substituted by

$$
\widetilde{K}_{1}(t)=\gamma(t)+\operatorname{ess} \sup _{\Omega}\left(\frac{m_{t}}{(m+1)^{2}}\right)
$$

and the coefficient $K(t)$ given by the formula

$$
\widetilde{K}(t)=\int_{0}^{t}\left(\gamma(\tau)+\operatorname{ess} \sup _{\Omega}\left(\frac{m_{t}}{(m+1)^{2}}\right)\right) d \tau .
$$

The differential inequality for the energy function $\widetilde{S}(t)=\frac{\mathrm{e}^{-\widetilde{K}(t)}}{M} \int_{\Omega}|v|^{m(x, t)+1} d x$ takes the form

$$
\forall \text { a.e. } t \in(0, T) \quad \widetilde{S}^{\prime}(t)+\frac{1}{2} \widetilde{D}(t) \widetilde{S}^{\nu^{+}(t)}(t) \leq \widetilde{\phi}(t), \quad \widetilde{S}(0)=\frac{1}{M} \int_{\Omega}\left|v_{0}\right|^{m_{0}+1} d x
$$

with the coefficient and the right-hand side

$$
\widetilde{D}(t)=C \mathrm{e}^{-\widetilde{K}(t)\left(1-\nu^{+}(t)\right)}, \quad \widetilde{\phi}(t)=\frac{l \mathrm{e}^{-\widetilde{K}(t)}}{M} \int_{\Omega} g(z) d x .
$$

The conclusions about the possibility of the finite-time extinction of the solution follow from the proofs of Theorems 6.1-6.2. 


\section{Large time behavior}

The results of Section 6 refer to the cases when either $\nu^{+}(t) \in\left(0, \nu_{0}\right)$ with $\nu_{0}<1$, or $\nu^{+}(t)$ is monotone increasing and tends to the critical value $\nu_{0}=1$ as $t \rightarrow \infty$. If neither of these conditions is fulfilled, the analysis of the differential inequality for the energy function leads to the following results.

Theorem 7.1. Let the conditions of Theorem 6.1 be fulfilled with $g \equiv 0$ in $Q_{T}$ and $v$ be a bounded energy solution of problem (1.1). Let $\nu^{+}(t):[0, \infty) \mapsto\left(0, \nu^{+}\right]$be the function defined in (4.2) if $b^{+}<0$, and in (4.6) if $b^{+}=0$.

1. If $\nu^{+}=1$, then

$$
\Sigma(t) \equiv \int_{\Omega}|v|^{m(z)+1} d x \leq \Sigma(0) \exp \left(K(t)-\frac{1}{2} C \int_{0}^{t}\left(\Sigma(0) \mathrm{e}^{K(s)}\right)^{\nu^{+}(s)-1} d s\right)
$$

with the function $K(t)$ from (5.7) and the constant $C$ from (5.6).

2. If $\nu^{+}>1$, then

$$
\Sigma(t) \leq \frac{\Sigma(0) \mathrm{e}^{K(t)}}{\left(1+\frac{1}{2} C\left(\nu^{+}-1\right) \int_{0}^{t}\left(\Sigma(0) \mathrm{e}^{K(s)}\right)^{\nu^{+}(s)-1} d s\right)^{\frac{1}{\nu^{+}-1}}} .
$$

3. If $\nu^{+}<1$, then

$$
\Sigma(t) \leq \Sigma(0) \max \left\{0,\left(1-\frac{1}{2} C\left(1-\nu^{+}\right) \int_{0}^{t}\left(\Sigma(0) \mathrm{e}^{K(s)}\right)^{\nu^{+}(s)-1} d s\right)^{\frac{1}{1-\nu^{+}}}\right\} .
$$

Proof. By Lemma 5.2 the energy function $S(t)=\frac{\Sigma(t)}{M} \mathrm{e}^{-K(t)}$ satisfies the homogeneous differential inequality (5.13). Since $S \in[0,1]$, we may continue (5.13) as follows:

$$
\forall \text { a.e. } t \in(0, T) \quad S^{\prime}+\frac{1}{2} C \mathrm{e}^{-K(t)\left(1-\nu^{+}(t)\right)} S^{\nu^{+}} \leq 0 .
$$

The assertions follow now by the direct integration of the last inequality.

Corollary 7.1. The large time behavior of energy solutions of problem (6.7) is also described by the estimates of Theorem 7.1, but with the coefficient $K(t)$ substituted by $\widetilde{K}(t)$ - see Subsection 6.3 .

\section{Acknowledgements}

The first author was partially supported by the FCT, Financiamente Base (Nos.2008ISFL-1-209, 2008-ISFL-1-297) Portugal and by the Research Project PTDC/ MAT/ 110613/ 2009, FCT, Portugal and by the Research Project MTM2011-26119, MICINN, Spain. The second author acknowledges the support of the Research Project MTM2010-18427, MICINN, Spain. 


\section{References}

[1] S. Antontsev, M. Chipot, And S. Shmarev, Uniqueness and comparison theorems for solutions of doubly nonlinear parabolic equations with nonstandard growth conditions. Commun. Pure Appl. Anal. 12, n.4 (2013) pp. 1527-1546.

[2] S. N. Antontsev, J. I. Díaz, and S. Shmarev, Energy Methods for Free Boundary Problems: Applications to Non-linear PDEs and Fluid Mechanics, Bikhäuser, Boston, 2002. Progress in Nonlinear Differential Equations and Their Applications, Vol. 48.

[3] S. N. Antontsev And S. I. Shmarev, Localization of solutions of anisotropic parabolic equations, Nonlinear Anal., 71 (2009), pp. e725-e737.

[4] - Blow-up of solutions to parabolic equations with non-standard growth conditions, Journal of Computational and Applied Mathematics, 234 (2010), pp. 2633-2645.

[5] — On the blow-up of solutions to anisotropic parabolic equations with variable nonlinearity, Tr. Mat. Inst. Steklova, 270 (2010), pp. 33-48.

[6] _ Vanishing solutions of anisotropic parabolic equations with variable nonlinearity, J. Math. Anal. Appl., 361 (2010), pp. 371-391.

[7] — Doubly degenerate parabolic equations with variable nonlinearity I: Existence of bounded strong solutions, Advances in Differential Equations, 17 (2012), pp. 1181-1212.

[8] M. Y. CHen, Blow-up for doubly degenerate nonlinear parabolic equations, Acta Math. Sin. (Engl. Ser.), 24 (2008), pp. 1525-1532.

[9] L. Diening, P. Harjulehto, P. HäStö, And M. Rüž̆ IčKa, Lebesgue and Sobolev Spaces with Variable Exponents, Springer, Berlin, 2011. Series: Lecture Notes in Mathematics, Vol. 2017, 1st Edition.

[10] I. Fragalà, F. Gazzola, and B. Kawohl, Existence and nonexistence results for anisotropic quasilinear elliptic equations, Ann. Inst. H. Poincaré Anal. Non Linéaire, 21 (2004), pp. 715-734.

[11] V. A. Galaktionov And J. L. VÁzquez, The problem of blow-up in nonlinear parabolic equations, Discrete Contin. Dyn. Syst., 8 (2002), pp. 399-433. Current developments in partial differential equations (Temuco, 1999).

[12] È. Mitidieri And S. I. Pokhozhaev, A priori estimates and the absence of solutions of nonlinear partial differential equations and inequalities, Tr. Mat. Inst. Steklova, 234 (2001), pp. 1-384.

[13] M. M. Porzio, Existence, uniqueness and behavior of solutions for a class of nonlinear parabolic problems, Nonlinear Anal., 74 (2011), pp. 5359-5382.

[14] J. RÁkosník, Some remarks to anisotropic Sobolev spaces. II, Beiträge Anal., (1980), pp. 127-140 (1981). 
[15] A. A. Samarskit, V. A. Galaktionov, S. P. Kurdyumov, and A. P. Mikhailov, Blow-up in quasilinear parabolic equations, vol. 19 of de Gruyter Expositions in Mathematics, Walter de Gruyter \& Co., Berlin, 1995. Translated from the 1987 Russian original by Michael Grinfeld and revised by the authors.

[16] M. Troisi, Teoremi di inclusione per spazi di Sobolev non isotropi, Ricerche Mat., 18 (1969), pp. 3-24.

[17] M. Tsutsumi, Existence and nonexistence of global solutions for nonlinear parabolic equations, Publ. Res. Inst. Math. Sci., Kyoto University. Research Institute for Mathematical Sciences. Publications, 8 (1972), pp. 211-229. 